\title{
Corticotropin-releasing factor induces immune escape of cervical cancer cells by downregulation of NKG2D
}

\author{
HYUNKEUN SONG ${ }^{1 *}$, HYUNJIN PARK ${ }^{1 *}$, GABIN PARK $^{1}$, YEONG SEOK KIM ${ }^{1}$, HYUN-KYUNG LEE ${ }^{2}$, \\ DONG-HOON JIN ${ }^{3}$, HYUNG-SIK KANG ${ }^{4}$, DAE-HO CHO ${ }^{5}$ and DAEYOUNG HUR ${ }^{1}$ \\ ${ }^{1}$ Department of Anatomy and Research Center for Tumor Immunology, Inje University College of Medicine;
${ }^{2}$ Department of Internal Medicine, Inje University Busan Paik Hospital, Busan 614-735; ${ }^{3}$ Institute for Innovative
Cancer Research, College of Medicine, University of Ulsan, Asan Medical Center, Seoul 138-736;
${ }^{4}$ School of Biological Sciences and Technology, Chonnam National University, Buk-gu, Gwangju 500-757;
${ }^{5}$ Department of Life Science, Sookmyung Women's University, Yongsan-ku, Seoul 140-742, Republic of Korea
}

Received February 21, 2014; Accepted April 23, 2014

DOI: $10.3892 /$ or.2014.3191

\begin{abstract}
Corticotropin-releasing factor (CRF), a coordinator of the body's responses to stress, is found in various cancer tissues and cell lines. However, the exact abilities of CRF to manipulate natural killer (NK) cells during immune response have not been studied. NKG2D is an activating receptor that is expressed on most $\mathrm{NK}$ and $\mathrm{CD}^{+} \mathrm{T}$ cells. MHC class I-related chain A (MICA) and UL16-binding protein (ULBP) 1, 2 and 3 are well-known ligands for NKG2D. In the present study, we reported our findings regarding the role of CRF in cervical cancer cell survival. Human cervical cancer cell line, HeLa cells, had significantly higher intracellular expression of UL16-binding protein 2 (ULBP2) following CRF treatment but had only slightly increased surface expression of ULBP2. Notably, MMPi (pan-metalloproteases inhibitor) blocked the release of ULBP2 molecules from the surface of HeLa cells. Furthermore, incubating NK cells with culture supernatants from CRF-treated HeLa cells, which contained soluble NKG2D ligand, reduced NK cell activity by decreasing surface expression of NKG2D. Collectively, downregulation of NKG2D by CRF-induced soluble NKG2D ligand provides a potential mechanism by which cervical cancer cells escape NKG2D-mediated attack under stress conditions.
\end{abstract}

\section{Introduction}

Corticotropin-releasing factor (CRF) was originally reported as a major hypothalamic mediating factor produced in response

Correspondence to: Professor Daeyoung Hur, Department of Anatomy and Research Center for Tumor Immunology, Inje University College of Medicine, 633-165 Kaekum-2-dong, Jin-gu, Busan 614-735, Republic of Korea

E-mail: dyhur@inje.ac.kr

*Contributed equally

Key words: corticotropin-releasing factor, NKG2D ligand, immune escape, natural killer cells to stress. The function of CRF in the homeostatic response to stress is well-characterized. The CRF system, consisting of CRF and CRF receptor (CRFR), has been identified in the cardiovascular, reproductive and gastrointestinal systems. CRF systems have been found in the immune system and are shown to possess immunomodulatory properties. Several studies have reported that $\mathrm{CRF}$ and its receptors have been found in many types of tumors (1-3). Urocortin, a CRF family member, was detected in various tumors, including pituitary adenoma, prostatic carcinoma and endometrial carcinoma (4-6). Notably, many studies report that $\mathrm{CRF}$ has both pro- and anticancer activity. Graziani et al revealed that CRF inhibited the proliferation of endometrial adenocarcinoma cells and human mammary cancer cells (7). CRF also inhibits proliferation of melanoma cells through mediation of CRFR1 (8). In contrast, other evidence suggests that CRF can promote cancer malignancy (9). Yang et al observed that CRF promoted the migration of melanoma cells via the ERK pathway (10). CRF can protect cancer cells from pro-apoptotic signals blocking activation of pro-caspase-3 (11). However, the cause of these opposing effects in tumors is not well understood.

Natural killer (NK) cells can recognize and lyse virusinfected cells and tumor cells (12). NK killing activity against tumor cells is a process that results from counterbalance between inhibitory and stimulatory signals. Specifically, NKG2D (activating receptor) mediates NK cell activation by overcoming the inhibitory signals from self-recognition (13). Moreover, NKG2D ligand is expressed on tumor cells and is induced by danger signals such as cellular stress and virus infection, suggesting that NKG2D ligands are potentially useful therapeutic targets for eliminating tumor- and virusinfected cells using NK cells (14-17). However, some studies have reported that tumor cells can produce soluble forms of NKG2D ligands that lead to downregulated surface NKG2D expression on NK and CTL cells (18-20). Salih et al observed that soluble NKG2D ligands secreted by tumor cells downregulate surface expression of NKG2D on NK cells and lead to NK cell malfunction. Moreover, we previously reported that soluble ULBP2 produced by gastric cancer inhibits function of NK cells through downregulation of NKG2D expression (21-23). 
In the present study, we investigated whether expression of NKG2D ligands is regulated by CRF in human cervical cancer cell lines, and we also defined the functional consequences of CRF-regulated NKG2D ligand production in cervical cancer cells following interaction between tumor and NK cells.

\section{Materials and methods}

Cell culture and reagents. Human cervical carcinoma cell line, HeLa, human alveolar basal epithelial cells, A549, and human leukemia cells, NK92 MI, were purchased from the American Type Culture Collection (ATCC; Manassas, VA, USA). HeLa and A549 cells were cultured in RPMI-1640 medium supplemented with $10 \%$ heat-inactivated fetal calf serum. NK92 MI cells were cultured in $\alpha$ modification of Minimum Essential Medium Eagle ( $\alpha$-MEM) supplemented with $2 \mathrm{mM}$ L-glutamine, $0.2 \mathrm{mM}$ inositol, $20 \mathrm{mM}$ folic acid, $12.5 \%$ FBS and $12.5 \%$ horse serum. Anti-NKG2D antibodies were purchased from R\&D Systems (Minneapolis, MN, USA). 1-[6-(17-3-methoxyestra-1,3,5(10)-trien-17-yl)amino) hexyl]-1H-pyrrole-2,5-dione (U73122) was purchased from Calbiochem (La Jolla, CA, USA). CRF and 1,10-phenanthroline (pan-matrix metalloprotease inhibitor) were purchased from Sigma-Aldrich (St. Louis, MO, USA).

Reverse transcription-polymerase chain reaction (RT-PCR). Total RNA was extracted from tumor cells using an RNeasy kit (Qiagen, Valencia, CA, USA). cDNA was prepared and used for RT-PCR with the following primers: human ULBP2 primer (sense, 5'-CTGCAGGTAAGGATGTCTTGTGAG-3' and antisense, 5'-TGAGGGTGGTGGCTATGGC-3', product 350 bp); human CRF (sense, 5'-CAACTTTTTCCGCGTGT TGCT-3' and antisense, 5'-ATGGCATAAGAGCAGCGC TAT-3', product $360 \mathrm{bp}$ ); human CRFR1 primer (sense, 5'-CAC TACCATGTTGCAGTCATC-3' and antisense, 5'-CGAACA TCCAGAAGAAGTTGG-3', product 300 bp); CRFR2 primer (sense, 5'-TCGTCAACTACCTGCGCCAC-3' and antisense, 5'-GTCATTAGGATCCTGACGATGT-3', product 522 bp). Cycling conditions for ULBP2, CRF, CRFR1 and CRFR2 were $1 \mathrm{~min}$ at $94^{\circ} \mathrm{C}, 1 \mathrm{~min}$ at $60^{\circ} \mathrm{C}$, and $1 \mathrm{~min}$ at $72^{\circ} \mathrm{C}$ for 32 cycles. $\beta$-actin was amplified as a control using sense primer, 5'-GTGGGGCGCCCCAGGCACCA-3' and antisense primer, 5'-CTCCTTAATGTCACGCACGATTTC-3', product $200 \mathrm{bp}$. Cycling conditions for $\beta$-actin were $1 \mathrm{~min}$ at $94^{\circ} \mathrm{C}$, $1 \mathrm{~min}$ at $55^{\circ} \mathrm{C}$ and $1 \mathrm{~min}$ at $72^{\circ} \mathrm{C}$ for 25 cycles. The PCR products were analyzed by EtBr-stained $1.2 \%$ agarose gel subjected to electrophoresis.

Flow cytometry analysis. Surface antigen FACS analysis was performed to detect NKG2D ligands on tumor cell lines and to detect NKG2D in NK cells. Briefly, cells were washed twice with ice-cold phosphate-buffered saline (PBS) and then incubated with mouse anti-human MHC class I-related chain A (MICA) monoclonal antibody, anti-human ULBP monoclonal antibodies (anti-ULBP1, anti-ULBP2 and anti-ULBP3) or anti-NKG2D antibody (both from R\&D Systems) for $30 \mathrm{~min}$ on ice. After two washes, cells were incubated with an appropriate FITC-conjugated secondary antibody diluted in PBS for 30 min on ice. Intracellular FACS analysis was performed to detect intracellular levels of NKG2D ligands in cervical cancer cells. Cells were washed twice with ice-cold PBS containing $0.05 \%$ BSA and $0.02 \%$ sodium azide (PBS-BSA), and fixed in $2 \%$ paraformaldehyde in PBS for $15 \mathrm{~min}$ on ice. The cells were then washed once in cold PBS-BSA and resuspended in PBS containing $0.1 \%$ saponin and $0.05 \%$ sodium azide (permeabilization buffer) for $15 \mathrm{~min}$, followed by incubation with an anti-NKG2D ligand antibody for $30 \mathrm{~min}$ on ice. After two washes, cells were incubated with an appropriate FITCconjugated secondary antibody in permeabilization buffer for 30 min on ice. Samples were analyzed using a FACSCalibur flow cytometer (BD Biosciences).

Cytotoxicity assays. NK cell cytotoxic activity was determined by calcein-AM assays (24). K562 target cells were washed twice with PBS and incubated with $5 \mathrm{mM}$ calcein-AM (Molecular Probes) in serum-free RPMI medium for $10 \mathrm{~min}$ at $37^{\circ} \mathrm{C}$. Labeled target cells were distributed into U-bottom microtiter plates at a concentration of $1 \times 10^{4}$ cells/well. Effector cells were added at various effector cell to target cell (E:T) ratios in quadruplicate. Target cells in complete RPMI medium alone were used to determine spontaneous calcein-AM retention. Maximal lysis was determined by solubilizing three wells of target cells in lysis buffer (0.1\% Triton X-100). After incubation for $5 \mathrm{~h}$, the assays were analyzed using a fluorescence reader. Percent specific cytotoxicity was calculated as follows: $\%$ Cytotoxicity $=[$ (retention of experimental well - retention of spontaneous well)/(retention of maximal lysis well - retention of spontaneous well)] x 100.

\section{Results}

CRF enhances expression of ULBP2 in cervical cancer cells. To evaluate expression of NKG2D ligands in cervical cancer cells, surface and intracellular expression of MICA and ULBP was analyzed (Fig. 1A). HeLa cells showed surface expression of MICA and ULBP2, but not ULBP1 and ULBP3. Intracellular expression of all NKG2D ligands was observed within HeLa cells by intracellular FACS staining analysis.

We next examined expression of CRF and the CRF receptor system in HeLa cells. A549 cells, which are known to express CRF, CRF receptor 1 and 2, were found to be positive for CRF and CRF receptor system by RT-PCR analysis (Fig. 1B, left panel). CRF and CRF receptor 2 genes were expressed in HeLa cells, but CRF receptor 1 was not detected by RT-PCR analysis (Fig. 1B, right panel). Several studies have reported that CRF and CRF receptors are expressed in many human cancers. We hypothesized that $\mathrm{CRF}$ and the CRF receptor system are involved in the response of stress associated NKG2D ligand expression in cervical cancer cells. To investigate the ability of CRF to alter NKG2D ligand expression on cervical cancer cells, we analyzed the intracellular level of NKG2D ligand in HeLa cells after treatment with CRF. We found that intracellular level of ULBP2 was strongly induced by CRF (Fig. 1C). However, other NKG2D ligands were not induced by CRF treatment (data not shown). Notably, ULBP2 surface expression was slightly induced in cervical cancer cells after treatment with CRF (Fig. 1C). These data suggest that production of CRF in HeLa cells may potentially induce ULBP2 expression. 


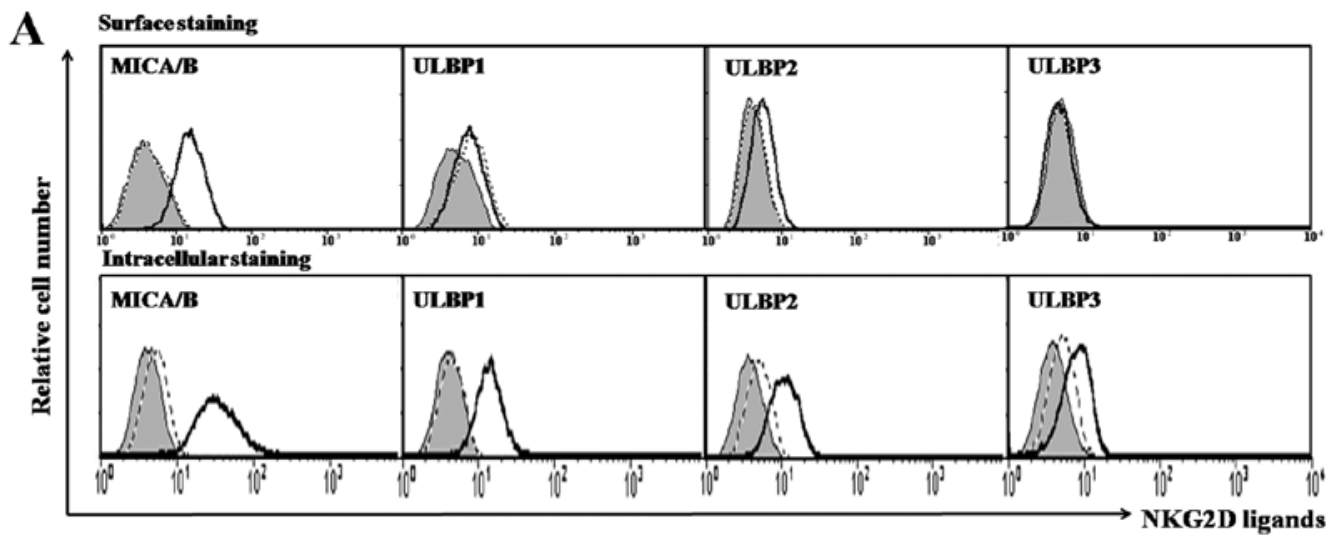

B
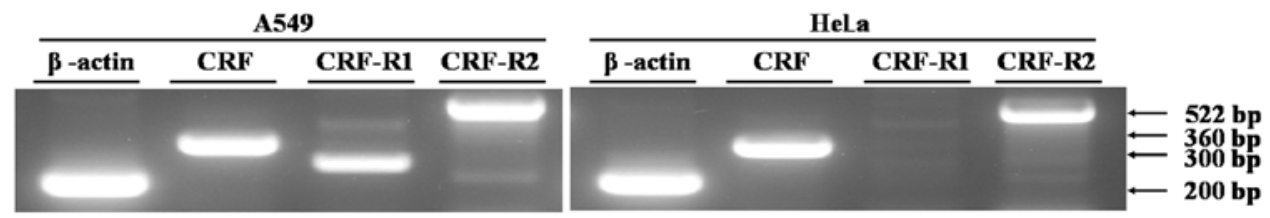

C ULBP2 Surface staining

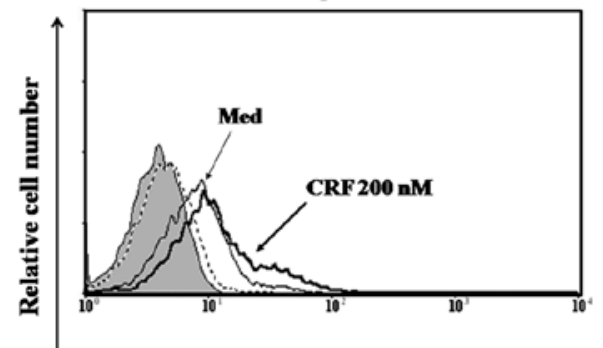

ULBP2 intracellularstaining

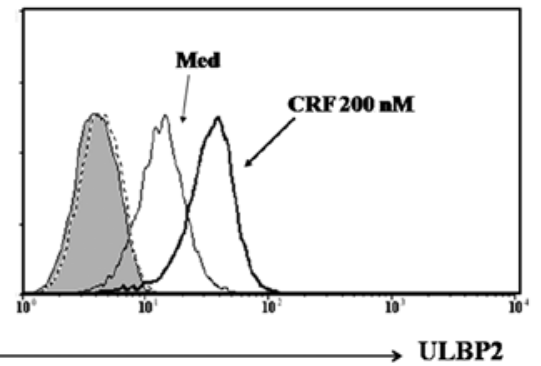

Figure 1. Expression of NKG2D ligands by cervical cancer cell lines. (A) Surface and intracellular staining of NKG2D ligand proteins. Human cervical cancer cells were stained with control normal mouse IgG (iso) or anti-MICA, anti-ULBP1, anti-ULBP2 and anti-ULBP3 antibodies and analyzed by flow cytometry. In the histograms, the gray filled line represents the unstained control. Dotted-line and thick-line histograms represent the isotype control and anti-NKG2D ligand mAb-treated cells, respectively. A representative result of three experiments performed is presented. (B) RT-PCR analysis of CRF, CRFR1 and CRFR2 mRNA from HeLa and A549 cells (positive expression of CRF/CRF receptor system in cells). RT-PCR was performed using RNA isolated from naïve cells. PCR products were analyzed by $1.5 \%$ agarose-gel electrophoresis. (C) CRF induced expression of ULBP2. Cervical cancer cells (HeLa) were incubated in the presence of CRF (200 nM) for $48 \mathrm{~h}$ and ULBP2 expression was analyzed by flow cytometry. Scattergrams on the left represent surface expression and those on the right represent intracellular expression.

To further confirm our findings, we evaluated the effects of various concentrations of CRF on ULBP2 expression in HeLa cells after treatment for 48 h. As shown in Fig. 2A and B, CRF treatment significantly induced intracellular expression of ULBP2 in a dose-dependent manner, but surface expression was not induced. As shown in Fig. 2C, ULBP2 mRNA expression was markedly induced by CRF in a dose-dependent manner.

Regulation of surface ULBP2 expression in cervical cancer. We observed that ULBP2 expression was markedly induced intracellularly following CRF treatment, but was not induced at the surface level (Fig. 2). We therefore addressed the question of whether CRF-induced ULBP2 could be expressed at surface level. ULBP 1, 2 and 3 are known to have a GPI-linked molecule, which can be released by phosphatidylinositolspecific phosphase C (PI-PLC). Moreover, Waldhauer and Steinle showed that ULBP2 can be released from tumor cells by metalloprotease (25). Based on this evidence, we investigated the effect of treatment with U73122 (PI-PLC inhibitor) and MMPi (pan-metalloprotease inhibitor) on ULBP2 surface expression following CRF treatment. After treatment with $5 \mu \mathrm{M}$ MMPi, CRF-treated HeLa cells showed strongly enhanced expression of ULBP2 compared with vehicle control-treated cells (Fig. 3A). In contrast, HeLa cells treated with PI-PLC inhibitor did not have enhanced surface expression of ULBP2 following CRF treatment. These data suggest that PI-PLC is not involved in the release of the soluble form of ULBP2 on CRF-treated HeLa cells.

Functional ability of soluble ULBP2 secreted from CRF-treated HeLa cells. In the present study, we observed that ULBP2 was expressed on the cell surface of HeLa cells following treatment with $\mathrm{CRF}$ and was released from the cell by metalloprotease. To determine the functions of soluble ULBP2, we treated NK cells with culture supernatant from CRF-treated HeLa cells. As shown in Fig. 4, we found that NK cells treated with CRF-treated HeLa cell culture supernatant for $48 \mathrm{~h}$ displayed significantly reduced activity in contrast to untreated NK cell control cells or NK cells treated directly with CRF. These results suggest that production of CRF from cervical cancer cells can reduce NK cell function via the NKG2D-mediated pathway. 

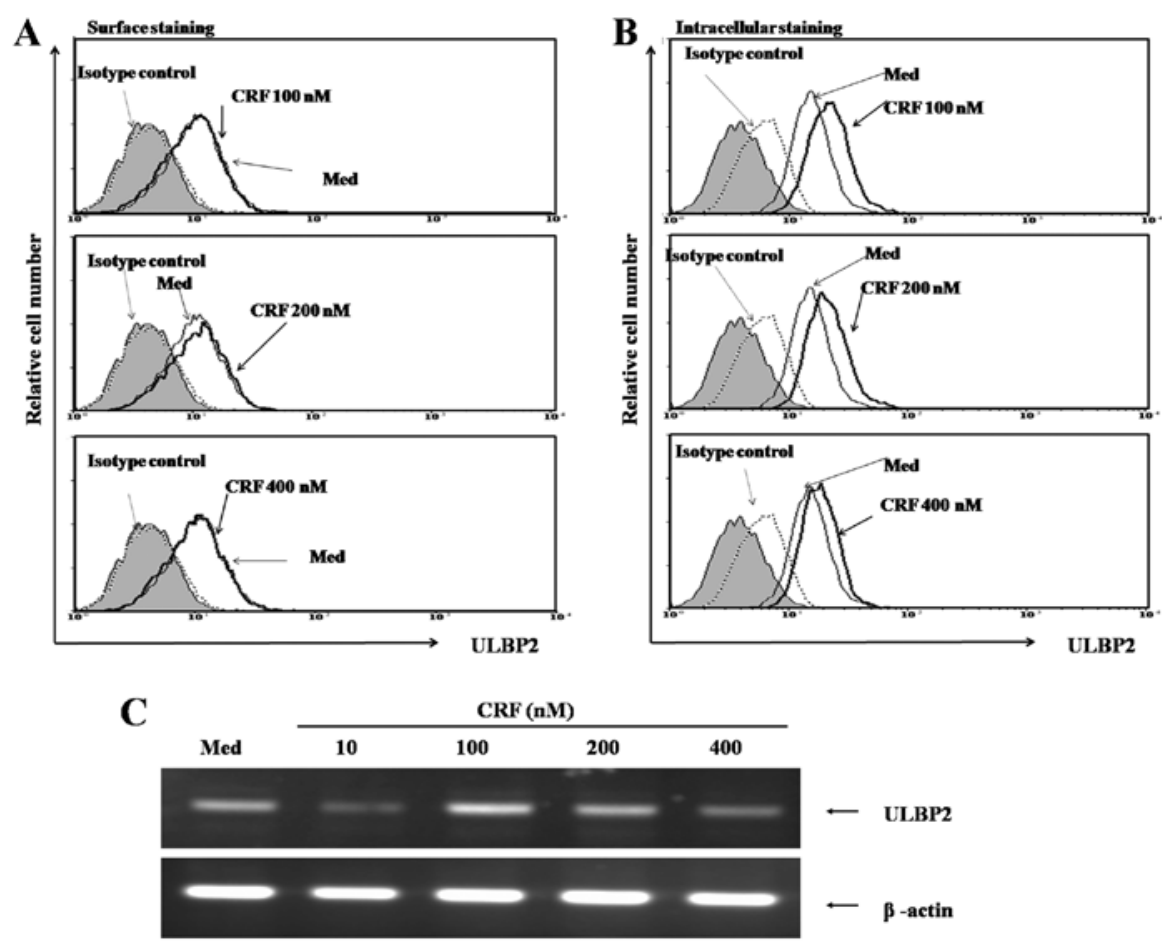

Figure 2. Expression of ULBP2 on cervical cancer cell lines following treatment with CRF. (A) Surface staining of ULBP2 protein. HeLa cells were incubated with various concentrations of CRF for $48 \mathrm{~h}$; subsequently, cells were washed with cold PBS. Cells were then stained with normal mouse IgG (iso) or anti-ULBP2 antibody and analyzed by flow cytometry. In the histograms, the gray filled line represents unstained control. A representative result of three experiments performed is presented. (B) Intracellular staining of ULBP2 protein after treatment with various concentrations of CRF. Human cervical cancer cells were analyzed for intracellular concentration of ULBP2 protein as described in Materials and methods. In the histograms, the gray filled line represents the unstained control. (C) RT-PCR analysis of ULBP2 mRNA in cervical cancer cell lines after treatment with various concentrations of CRF. RT-PCR was performed using primers for ULBP2 or $\beta$-actin. PCR products were analyzed by $1 \%$ agarose-gel electrophoresis.

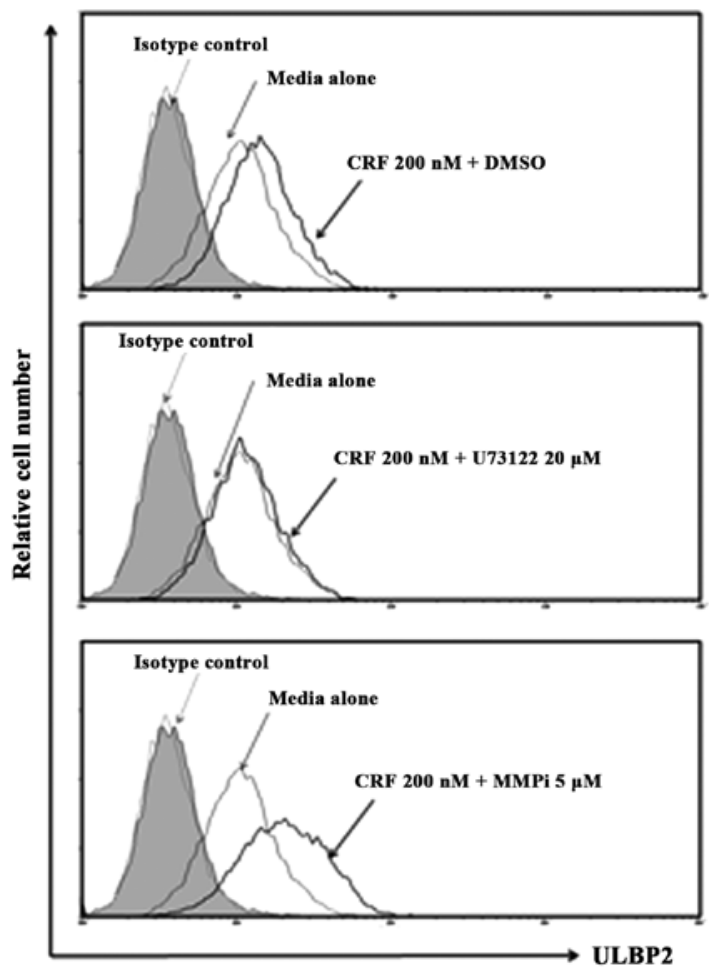

Figure 3. Regulation of surface expression of ULBP2 by U73122 (PI-PLC inhibitor) and MMPi (pan-metalloprotease inhibitor). Cervical cancer cells were pre-incubated with $20 \mu \mathrm{M} \mathrm{U} 73122$ and $5 \mu \mathrm{M} \mathrm{MMPi}$. After $1 \mathrm{~h}$, cells were incubated with $200 \mathrm{nM} \mathrm{CRF}$ for $48 \mathrm{~h}$; cells were subsequently analyzed by flow cytometric analysis. In the histograms, the gray filled line represents the unstained control. A representative result of three experiments is presented.

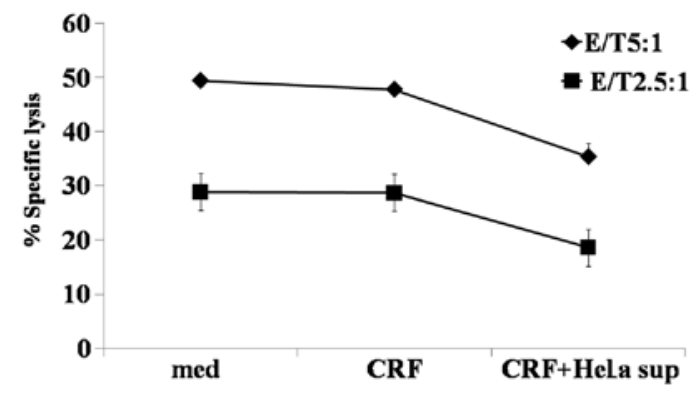

Figure 4. Functional activity of culture supernatants from CRF-treated human cervical cancer cells. Cytotoxic activity of NK92MI cells against K562 cells was analyzed after pre-incubating NK92MI cells $\left(2 \times 10^{6}\right.$ cells/well $)$ with complete media (med) and directly treated with $200 \mathrm{nM} \mathrm{CRF}(\mathrm{CRF})$ and CRF-treated HeLa supernatant $(25 \%$, v/v) for $72 \mathrm{~h}$. Two-day-culture supernatants from cancer cells were used for pre-incubation experiments. Results are representative of three independent experiments.

\section{Discussion}

The present study demonstrated the inhibitory function of CRF in NKG2D-mediated NK killing activity against cervical cancer cells by inducing release of soluble NKG2D ligand from cancer cells and suggests the possible role of cancer produced $\mathrm{CRF}$ as an immunomodulator between immune cells and cancer cells. Corticotrophin-releasing factor (CRF) was originally identified as a regulator of the stress response in hypothalamic-pituitary-adrenal axis (26). CRF and the CRF homologues urocortins (UCN) have previously been detected 
in many types of cancer, such as endometrial carcinoma, prostatic carcinoma and pituitary adenoma (4-6). Reubi et al showed that the CRF receptors or CRF are expressed in various human cancers (2). Klimaviciute et al reported that mRNA and proteins of CRF receptors are expressed in human cervix and $\mathrm{CRF}$ is detected in cervical tissue, indicating that the cervix is a target for the action of CRF (27). Moreover, Minas et al observed that expression of CRF and CRF receptors detected in ovarian cancer in situ and corresponding secretion of CRF modulate survival of ovarian cancer cells (28). This evidence suggests that CRF and its receptor systems have critical functions in the regulation of cancer cell progression. In this study, we observed that CRF and CRF receptor 2 were strongly expressed in cervical cancer cells (Fig. 1). As shown in Fig. 1, exogenous treatment with CRF can induce intracellular levels of an NKG2D ligand (ULBP2) in cervical cancer cells. Moreover, we found that CRF-induced ULBP2 was released to the surface of cancer cells by metalloprotease (Fig. 3). Based on these results, we speculate that CRF produced by cancer cells may be involved in evasion of cervical cancer through downregulation of NKG2D in NK cells.

The NKG2D/NKG2D ligand system is particularly important to immune surveillance of tumors since expression of NKG2D ligands is induced by carcinogens and stress such as genotoxic stress (29-31). Human NKG2D ligands consist of two families: MHC class I-related molecules (MICs) and UL16-binding proteins (ULBPs) (16). We originally hypothesized that CRF induces MICA/B in cervical cancer cells since stress-inducible MICA/B is expressed in certain epithelial origin tumors following stress signals. In addition, several studies have revealed that ULBP1 is preferentially induced over other ULBPs in certain cancer cells $(32,33)$. In contrast to our expectations, we found that CRF treatment elevated levels of intracellular ULBP2 in cervical cancer cells. Moreover, intracellular expression of ULBP2 increased in a dose-dependent manner following CRF treatment. The ability of CRF to alter expression of various NKG2D ligands in various cancer cells may be an interesting topic for further studies.

It is well described that soluble forms of NKG2D ligands can be used by cancer cells to escape immunosurveillance of immune effector cells. Specifically, shedding of MICA has been shown to be an immune escape mechanism of tumors via downregulation of NKG2D on immune effector cells (34-36). Some studies have observed soluble forms of ULBP2 in certain cancer cells that are secreted by phospholipase since the ULBP2 structure consists of GPI-anchored molecule at the cell surface (37). However, we found that surface expression of ULBP2 does not increase following treatment with phospholipase inhibitor (U73122), but treatment with metalloprotease significantly induced ULBP2 surface expression, suggesting that ULBP2 cleavage was performed by metalloprotease (Fig. 3). Some studies have reported that cleavage of MICA and ULBP2 on cancer cells was significantly inhibited by metalloprotease inhibitor $(20,25,38)$. In addition, several studies have also reported that CRF can induce expression of metalloprotease in cervical tissue $(39,40)$. These results suggest that proteolytic release of ULBP2 on cancer cells occurs through CRF-induced metalloprotease during cancer immune escape. This aspect should be investigated in future studies.
In conclusion, ULBP2 is expressed and released from cervical cancer cells by CRF, which regulates NKG2D expression in NK cells. The stability of cell surface expression and amount of ULBP2 released seems to be related to NK susceptibility. Mechanisms to modulate surface expression and soluble ULBP2 may provide another novel concept for immunotherapy to improve the function of $\mathrm{T}$ and NK cells in killing cervical cancer cells under stress conditions.

\section{Acknowledgements}

This study was supported by a grant from the National R\&D Program for Cancer Control, Ministry for Health, Welfare and Family Affairs, Republic of Korea (0920060), and by the Basic Science Research Program through the National Research Foundation of Korea (NRF) funded by the Ministry of Education, Science and Technology (2009-0073592).

\section{References}

1. Ciocca DR, Puy LA, Fasoli LC, et al: Corticotropin-releasing hormone, luteinizing hormone-releasing hormone, growth hormone-releasing hormone, and somatostatin-like immunoreactivities in biopsies from breast cancer patients. Breast Cancer Res Treat 15: 175-184, 1990.

2. Reubi JC, Waser B, Vale W and Rivier J: Expression of CRF1 and CRF2 receptors in human cancers. J Clin Endocrinol Metab 88: 3312-3320, 2003

3. Sato H, Nagashima Y, Chrousos GP, Ichihashi M and Funasak Y: The expression of corticotropin-releasing hormone in melanoma. Pigment Cell Res 15: 98-103, 2002.

4. Arcuri F, Cintorino M, Florio P, et al: Expression of urocortin mRNA and peptide in the human prostate and in prostatic adenocarcinoma. Prostate 52: 167-172, 2002.

5. Florio P, De Falco G, Leucci E, et al: Urocortin expression is downregulated in human endometrial carcinoma. J Endocrinol 190: 99-105, 2006.

6. Iino K, Sasano H, Oki Y, et al: Urocortin expression in human pituitary gland and pituitary adenoma. J Clin Endocrinol Metab 82: 3842-3850, 1997.

7. Graziani G, Tentori L, Muzi A, et al: Evidence that corticotropinreleasing hormone inhibits cell growth of human breast cancer cells via the activation of CRH-R1 receptor subtype. Mol Cell Endocrinol 264: 44-49, 2007.

8. Carlson KW, Nawy SS, Wei ET, et al: Inhibition of mouse melanoma cell proliferation by corticotropin-releasing hormone and its analogs. Anticancer Res 21: 1173-1179, 2001.

9. Arbiser JL, Karalis K, Viswanathan A, et al: Corticotropinreleasing hormone stimulates angiogenesis and epithelial tumor growth in the skin. J Invest Dermatol 113: 838-842, 1999.

10. Yang Y, Park H, Kim TS, Bang SI and Cho D: Enhancement of cell migration by corticotropin-releasing hormone through ERK1/2 pathway in murine melanoma cell line, B16F10. Exp Dermatol 16: 22-27, 2007.

11. Radulovic M, Hippel C and Spiess J: Corticotropin-releasing factor (CRF) rapidly suppresses apoptosis by acting upstream of the activation of caspases. J Neurochem 84: 1074-1085, 2003.

12. Trinchieri G: Biology of natural killer cells. Adv Immunol 47: 187-376, 1989.

13. Raulet DH: Interplay of natural killer cells and their receptors with the adaptive immune response. Nat Immunol 5: 996-1002, 2004.

14. Bahram S, Inoko H, Shiina T and Radosavljevic M: MIC and other NKG2D ligands: from none to too many. Curr Opin Immunol 17: 505-509, 2005.

15. Groh V, Steinle A, Bauer S and Spies T: Recognition of stressinduced MHC molecules by intestinal epithelial $\gamma \delta \mathrm{T}$ cells. Science 279: 1737-1740, 1998.

16. Raulet DH: Roles of the NKG2D immunoreceptor and its ligands. Nat Rev Immunol 3: 781-790, 2003.

17. Welte SA, Sinzger C, Lutz SZ, et al: Selective intracellular retention of virally induced NKG2D ligands by the human cytomegalovirus UL16 glycoprotein. Eur J Immunol 33: 194-203, 2003. 
18. Groh V, Wu J, Yee C and Spies T: Tumour-derived soluble MIC ligands impair expression of NKG2D and T-cell activation. Nature 419: 734-738, 2002.

19. Pende D, Rivera P, Marcenaro S, et al: Major histocompatibility complex class I-related chain A and UL16-binding protein expression on tumor cell lines of different histotypes: analysis of tumor susceptibility to NKG2D-dependent natural killer cell cytotoxicity. Cancer Res 62: 6178-6186, 2002.

20. Waldhauer I, Goehlsdorf D, Gieseke F, et al: Tumor-associated MICA is shed by ADAM proteases. Cancer Res 68: 6368-6376, 2008.

21. Salih HR, Antropius H, Gieseke F, et al: Functional expression and release of ligands for the activating immunoreceptor NKG2D in leukemia. Blood 102: 1389-1396, 2003.

22. Salih HR, Holdenrieder S and Steinle A: Soluble NKG2D ligands: prevalence, release, and functional impact. Front Biosci 13: 3448-3456, 2008.

23. Song H, Kim J, Cosman D and Choi I: Soluble ULBP suppresses natural killer cell activity via down-regulating NKG2D expression. Cell Immunol 239: 22-30, 2006.

24. Lichtenfels R, Biddison WE, Schulz H, Vogt AB and Martin R: CARE-LASS (calcein-release-assay), an improved fluorescencebased test system to measure cytotoxic T lymphocyte activity. J Immunol Methods 172: 227-239, 1994.

25. Waldhauer I and Steinle A: Proteolytic release of soluble UL16binding protein 2 from tumor cells. Cancer Res 66: 2520-2526, 2006.

26. Bale TL and Vale WW: CRF and CRF receptors: role in stress responsivity and other behaviors. Annu Rev Pharmacol Toxicol 44: 525-557, 2004.

27. Klimaviciute A, Calciolari J, Bertucci E, et al: Corticotropinreleasing hormone, its binding protein and receptors in human cervical tissue at preterm and term labor in comparison to nonpregnant state. Reprod Biol Endocrinol 4: 29, 2006.

28. Minas V, Rolaki A, Kalantaridou SN, et al: Intratumoral CRH modulates immuno-escape of ovarian cancer cells through FasL regulation. Br J Cancer 97: 637-645, 2007.

29. Cerwenka A and Lanier LL: Ligands for natural killer cell receptors: redundancy or specificity. Immunol Rev 181: 158-169, 2001 .
30. Diefenbach A and Raulet DH: Strategies for target cell recognition by natural killer cells. Immunol Rev 181: 170-184, 2001.

31. Lanier LL: NKG2D. J Biol Regul Homeost Agents 17: 338-340, 2003.

32. López-Soto A, Quiñones-Lombraña A, López-Arbesú R López-Larrea C, González S: Transcriptional regulation of ULBP1, a human ligand of the NKG2D receptor. J Biol Chem 281: 30419-30430, 2006.

33. Rohner A, Langenkamp U, Siegler U, Kalberer CP and Wodnar-Filipowicz A: Differentiation-promoting drugs up-regulate NKG2D ligand expression and enhance the susceptibility of acute myeloid leukemia cells to natural killer cell-mediated lysis. Leuk Res 31: 1393-1402, 2007.

34. Raffaghello L, Prigione I, Airoldi I, et al: Downregulation and/or release of NKG2D ligands as immune evasion strategy of human neuroblastoma. Neoplasia 6: 558-568, 2004.

35. Salih HR, Rammensee HG and Steinle A: Cutting edge: downregulation of MICA on human tumors by proteolytic shedding. J Immunol 169: 4098-4102, 2002.

36. Wu JD, Higgins LM, Steinle A, Cosman D, Haugk K and Plymate SR: Prevalent expression of the immunostimulatory MHC class I chain-related molecule is counteracted by shedding in prostate cancer. J Clin Invest 114: 560-568, 2004.

37. Onda H, Ohkubo S, Shintani Y, et al: A novel secreted tumor antigen with a glycosylphosphatidylinositol-anchored structure ubiquitously expressed in human cancers. Biochem Biophys Res Commun 285: 235-243, 2001.

38. Fernández-Messina L, Ashiru O, Boutet $\mathrm{P}$, et al: Differential mechanisms of shedding of the glycosylphosphatidylinositol (GPI)-anchored NKG2D ligands. J Biol Chem 285: 8543-8551, 2010.

39. Dubicke A, Akerud A, Sennstrom M, et al: Different secretion patterns of matrix metalloproteinases and IL-8 and effect of corticotropin-releasing hormone in preterm and term cervical fibroblasts. Mol Hum Reprod 14: 641-647, 2008.

40. Li W and Challis JR: Corticotropin-releasing hormone and urocortin induce secretion of matrix metalloproteinase-9 (MMP-9) without change in tissue inhibitors of MMP-1 by cultured cells from human placenta and fetal membranes. J Clin Endocrinol Metab 90: 6569-6574, 2005 